\title{
Influences of Lubricant Viscosity Ratio on Static Characteristics of Hydrostatic Thrust Bearing
}

\author{
Chuan-Chieh Liao, Sheng-Yen Hu, Chao-Ping Huang and Yuan Kang ${ }^{\mathrm{a}}$ \\ Chung Yuan Christian University, Department of Mechanical Engineering, 32023 Chung Li, Taiwan, R.O.C
}

\begin{abstract}
This paper uses numerical simulation to study the influences of viscosity ratio on the static characteristics of hydrostatic thrust bearing compensated by various restrictors. The design parameters of open type hydrostatic bearing include: effective area, supply pressure, restriction constants of restrictor and bearing pad, and specific value of average viscosity of the lubricant passing through restrictor and bearing pad film. The optimal design requirements of restrictors and hydrostatic bearing and the corresponding range of design parameters for excellent bearing characteristics can be obtained from the relationships between these design parameters together with their composed parameters and ratios, and the static load capacity and film stiffness and worktable displacement of hydrostatic bearing. Fixing the other design parameters and changing viscosity ratio, the simulation results depict that the lubricant viscosity during restrictor being greater than that in bearing pad film will make the load capacity decrease, but will improve the film static stiffness.
\end{abstract}

\section{Introduction}

Hydrostatic bearings according to their applications can be divided into thrust, radial and biaxial three kind bearing systems. Lubricating oil was pumped by an external pump through a restrictor into recess to form restriction pressure, then flew into the bearing clearance and provided bearing pad pressure to form a lubricating film supporting the load. The restrictors can apply constant compensations such as capillary, orifice and slot, or variable compensations such as pressure feedback driving membranetype and spool-type restrictors, or use one recess connected to one constant flow pump or valve to adjust supply pressure into working pressure by constant flow compensation.

Malanoski and Loeb [1] studied hydrostatic bearings using capillary, orifice or flow control valve as the pressure compensation elements. They investigated the influences of these three compensated types on the film stiffness of hydrostatic bearings, and found the stiffness using flow control valve will be the greatest, followed by using orifice, then using capillary. Wang [2] investigated the load capacity and film stiffness of hydrostatic slide, and exploded the influences of film thickness and slider clearance on worktable characteristics. Kang et al. [3] investigated the constant compensations. They utilized the equations of flow equilibrium to determine the worktable displacement with respect to the recess pressure. The influences of bearing pad and restrictor geometric design parameters on its static characteristics: load capacities and stiffness coefficients were investigated.

a Corresponding author: yk@cycu.edu.tw 
Nikolakopoulos and Papadopoulos [4] analytically and experimentally studied ER fluid lubricated journal bearings, and indicated ER fluid can be used to create "smart" journal bearings and vibration controllers can be constructed to control the dynamics and stability of the ER fluid lubricated bearings. Bouzidane and Thomas [5-6] studied the vibration control of an ER hydrostatic journal bearing. They found that the viscosity of a negative ER (NER) fluid will decrease with an electric field applied. They concluded that using NER fluid lubrication is very efficient when the force transmitted to the base must be reduced. Yu et al [7] analyzed the influence of rotational speed and viscosity for 3D temperature distribution. But they didn't discussed the influence of viscosity caused by temperature for the load capacity and static stiffness.

These previous studies of hydrostatic bearing applied electromagnetic rheological fluid as lubricant. When the local external magnetic field affected lubricant viscosity as it pass through at the same time, the characteristics of bearing have been changed. This is mainly caused by the difference between the viscosity of lubricant flowing through the restrictor and supporting film within the system. Therefore, this study investigated the influences of viscosity ratio of lubricant passing through restrictor to supporting film on the static characteristics and performances of hydrostatic bearing.

\section{Numerical formulations}

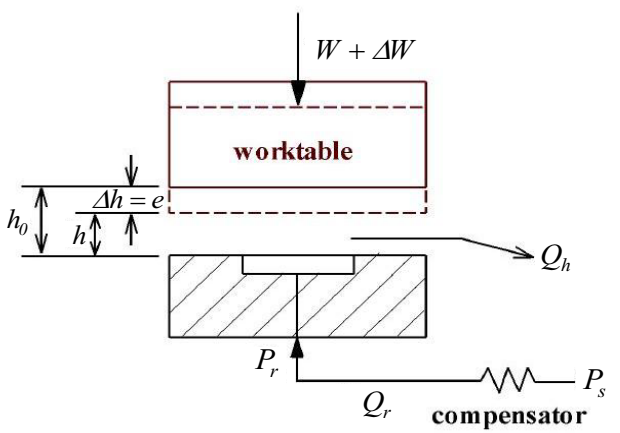

Figure 1. Open-type hydrostatic planar bearing

For an open-type hydrostatic bearing as shown in Fig. 1, the volumetric flow rate $Q_{h}$ out of bearing pad will be

$$
Q_{h}=\left(P_{r} h^{3} \gamma\right) /\left(12 \mu_{h}\right)
$$

where $P_{r}$ is the work pressure, $h$ is the film thickness, $\mu_{h}$ is the lubricant viscosity of oil film, and $\gamma$ denoted for dimensionless parameter of pad restriction that is a proportional constant of film flow due to pad geometry. Furthermore, dividing $\left(P_{s} h_{0}{ }^{3}\right) /\left(12 \mu_{r}\right)$ can obtain the dimensionless flow is:

$$
\bar{Q}_{h}=\frac{12 \mu_{r} Q_{h}}{P_{s} h_{0}^{3}}=\frac{P_{s}}{P_{r}} \frac{\mu_{r}}{\mu_{h}}\left(\frac{h}{h_{0}}\right)^{3} \gamma=\bar{P} \bar{\mu} \bar{h}^{3} \gamma
$$

where $\mu_{r}$ denotes the viscous coefficient of oil during compensator, $P_{s}$ is the system supply pressure, is the characteristic clearance of open-type hydrostatic bearing as the bearing pad and restrictor been designed, $\bar{\mu}$ is the viscosity ratio of lubricant in restrictor to recess $\left(\mu_{r} / \mu_{h}\right)$, and $\bar{h}=h / h_{0}$ is the film thickness ratio. The load capacity of an open-type hydrostatic bearing can be determined by $W=P_{r} A_{e f f}$, and its non-dimensional form is:

$$
\bar{W}=W /\left(P_{s} A_{e f f}\right)=\bar{P}_{r}
$$

where $A_{\text {eff }}$ is the effective area of bearing pad, which is smaller than the pad area, thus, a greater $A_{\text {eff }}$ is indicated for a larger recess proportion of bearing pad. The film static stiffness is defined by the load variation with respect to the displacement variation of worktable and expressed as 


$$
K=\frac{d W}{d e}=\frac{-d W}{d h}=\frac{-P_{s} A_{e f f}}{h_{0}} \frac{d \bar{P}_{r}}{d h}
$$

where $e=h_{0}-h$ is the displacement of worktable. Dividing Eq. (4) by $P_{s} A_{\text {eff }} / h_{0}$ gives the dimensionless film stiffness:

$$
\bar{K}=\frac{K h_{0}}{P_{s} A_{\text {eff }}}=\frac{d W}{d e} \frac{h_{0}}{P_{s} A_{\text {eff }}}=\frac{-d \bar{P}_{r}}{d \bar{h}}
$$

In order to make the relationship between the bearing design parameters and characteristic parameters generalized, the equations established with dimensionless parameters are adopted. Hence, according to Eqs. (3) and (5), the dimensionless load capacity $\bar{W}$ and static stiffness coefficient $\bar{K}$ can be obtained from design conditions respectively, and show that load capacity is proportional to $P_{s} A_{e f f}$ and static stiffness is proportional to $\left(P_{s} A_{e f f}\right) / h_{0}$.

\subsection{Constant flow compensation}

The constant flow compensation can be realized by giving constant flow for recess pressure varying to bear the load fluctuation, and the continuity equation can be expressed as:

$$
Q_{p}=\left(P_{r} h^{3} \gamma\right) /\left(12 \mu_{h}\right)=\text { constant }
$$

Assuming the design film thickness for constant flow compensation to be $h_{0}^{3}=\left(12 Q_{p} \mu_{r}\right) / P_{s}$, which gives a dimensionless form of Eq. (6) as below:

$$
\gamma \bar{\mu} \bar{P}_{r} \bar{h}^{3}=1
$$

Therefore, the unit dimensionless load capacity for an open-type hydrostatic bearing compensated by constant flow compensation will be

$$
\bar{W}=\bar{P}_{r}=\left(\gamma \bar{\mu} \bar{h}^{3}\right)^{-1}
$$

Differentiating equation (8) with respect to $\bar{h}$, then, substituting it into equation (5) gives the nondimensional film stiffness as:

$$
\bar{K}=\frac{-d \bar{P}_{r}}{d \bar{h}}=3\left(\gamma \bar{\mu} \bar{h}^{4}\right)^{-1}=3(\gamma \bar{\mu})^{1 / 3}\left(\bar{P}_{r}\right)^{4 / 3}
$$

where $\bar{h}=\left(\gamma \bar{\mu} \bar{P}_{r}\right)^{-1 / 3}$.

\subsection{Capillary compensation}

The flow rate through a capillary restrictor can be determined by:

$$
Q_{c}=\frac{\pi d_{c}^{4}}{128 \mu_{r} \ell_{c}}\left(P_{s}-P_{r}\right)=\frac{\kappa_{c}}{\mu_{r}}\left(P_{s}-P_{r}\right)
$$

where $d_{c}$ and $\ell_{c}$ are diameter and length of capillary, and $\kappa_{c}$ is the restriction constant of capillary. Therefore, the continuity equation of flow through capillary restrictor to bearing pad film is

$$
\frac{P_{r} h^{3}}{12 \mu_{h}} \gamma=\frac{\kappa_{c}}{\mu_{r}}\left(P_{s}-P_{r}\right)
$$

Letting the design film thickness of open-type bearing compensated by capillary to be $h_{0}^{3}=3 \pi d_{c}^{4} / 32 \ell_{c}=12 \kappa_{c}$, which gives a dimensionless form of equation (11) as below:

$$
\bar{P}_{r} \bar{h}^{3} \gamma \bar{\mu}=1-\bar{P}_{r}
$$

The dimensionless load capacity for capillary compensated bearing can be observed by 
rearranging equation (12) as following:

$$
\bar{W}=\bar{P}_{r}=\left(\gamma \bar{\mu} \bar{h}^{3}+1\right)^{-1}
$$

To observe the formulation of non-dimensional film stiffness, we first differentiate equation (13) with respect to $\bar{h}$, then, substituting it into equation (5) gives the final formulation as:

$$
\bar{K}=\frac{-d \bar{P}_{r}}{d \bar{h}}=\frac{3 \gamma \bar{\mu} \bar{h}^{2}}{\left(\gamma \bar{\mu} \bar{h}^{3}+1\right)^{2}}=3 \sqrt[3]{\gamma \bar{\mu} \bar{P}_{r}^{-1}\left(1-\bar{P}_{r}\right)^{2}}
$$

where $\bar{h}=\sqrt[3]{\left(\bar{P}_{r}^{-1}-1\right)(\gamma \bar{\mu})^{-1}}$

\subsection{Orifice compensation}

The flow through an orifice can be expressed by

$$
Q_{o}=c_{o} \frac{\pi d_{o}^{2}}{4} \sqrt{\frac{2\left(P_{s}-P_{r}\right)}{\rho}}=\kappa_{o} \sqrt{P_{s}-P_{r}}
$$

where $c_{o}$ is geometric factor of orifice, $d_{o}$ is orifice diameter, $\rho$ is oil density, and $\kappa_{o}=\left(c_{o} \pi d_{o}^{2}\right)$ $\times(\sqrt{8 \rho})^{-1}$ is restriction constant of orifice. Here, we assume the applied lubricant is an incompressible fluid and $\kappa_{o}$ is constant. Then, the flow rate through the orifice is proportional to $\kappa_{o}$. Therefore, the continuity equation of flow through orifice restrictor to bearing pad film is

$$
\left(P_{r} h^{3}\right) /\left(12 \mu_{h}\right)=\kappa_{o} \sqrt{P_{s}-P_{r}}
$$
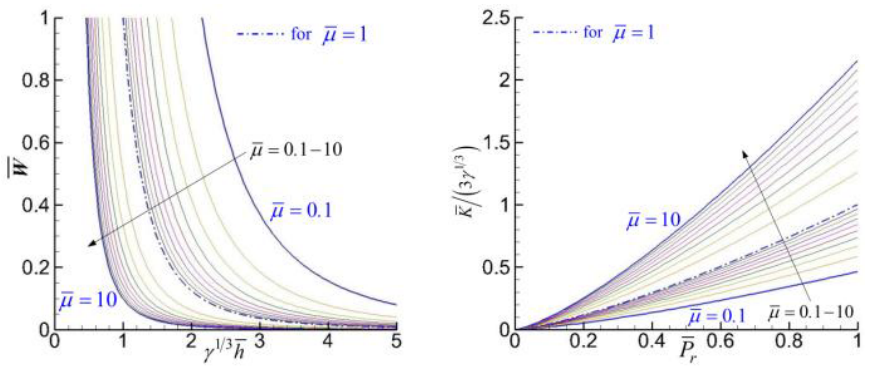

(a) constant flow compensation
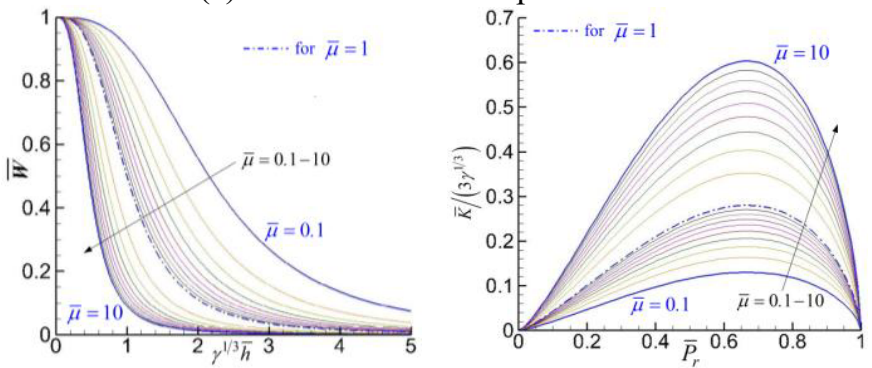

(b) Capillary compensation 

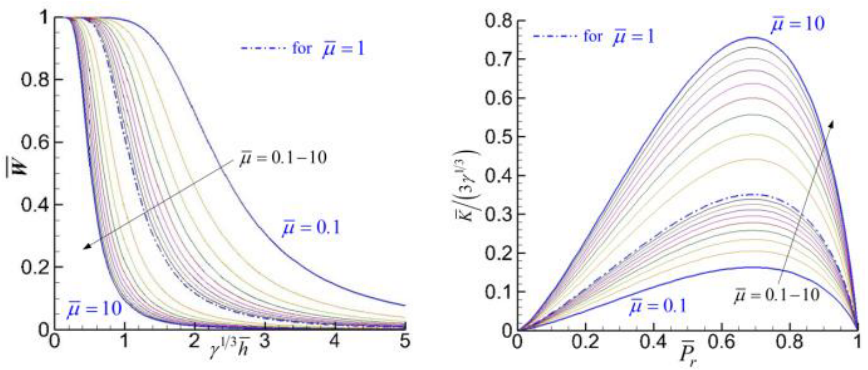

(c) Orifice compensation

Figure 2. $\bar{W}$ versus $\gamma^{1 / 3} \bar{h}$ (see left column) and $\bar{K} /\left(3 \gamma^{1 / 3}\right)$ versus $\bar{P}_{r}$ (see right column) for different $\bar{\mu}$ using different compensations of open-type hydrostatic bearing.

Setting the design film thickness of open-type bearing compensated by orifice to be $h_{0}^{3}=\sqrt{8} \pi d_{o}^{2} c_{o} \mu_{r} / \sqrt{P_{s} \rho}=12 \mu_{r} \kappa_{o} / \sqrt{P_{s}}$, which gives a dimensionless form of equation (16) as:

$$
\bar{P}_{r} \bar{h}^{3} \gamma \bar{\mu}=\sqrt{1-\bar{P}_{r}}
$$

The dimensionless load capacity for orifice compensated bearing can be observed by rearranging equation (18) as following:

$$
\bar{W}=\bar{P}_{r}=\frac{-1+\sqrt{1+4 \gamma^{2} \bar{\mu}^{2} \bar{h}^{6}}}{2 \gamma^{2} \bar{\mu}^{2} \bar{h}^{6}}
$$

To observe the formulation of non-dimensional film stiffness, we first differentiate equation (18) with respect to $\bar{h}$, then, substituting it into equation (5) gives the final formulation as:

$$
\bar{K}=\frac{-d \bar{P}_{r}}{d \bar{h}}=\frac{6 \gamma^{1 / 3} \bar{\mu}^{1 / 3}\left(1-\bar{P}_{r}\right)^{5 / 6} \bar{P}_{r}^{4 / 3}}{2-\bar{P}_{r}}
$$

where $\bar{h}=\left[\sqrt{1-\bar{P}_{r}}\left(\gamma \bar{\mu} \bar{P}_{r}\right)^{-1}\right]^{1 / 3}$.

\section{Results}

According to Eq. (8) for constant flow compensation, Eq. (13) for capillary restriction, and Eq. (18) for orifice restriction, the load capacity $\bar{W}$ is equal to the pressure ratio $\bar{P}_{r}$. The film thickness $\bar{h}$ is inversely proportional to the $\bar{\mu}^{1 / 3}$ under the same load, in other words, the $\bar{W}$ is lower as $\bar{\mu}$ being greater with the same film thickness. However, according to Eqs. (9), (14) and (19) for the relationship between static stiffness $\bar{K}$ and $\bar{P}_{r}$, the stiffness is proportional to $\bar{\mu}^{1 / 3}$. Plot $\bar{W}$ versus $\gamma^{1 / 3} \bar{h}$ and $\bar{K} /\left(3 \gamma^{1 / 3}\right)$ versus $\bar{P}_{r}$ for various $\bar{\mu}$ gives Fig. 2 . $\bar{W}$ will be smaller as $\bar{\mu}$ being greater with the same $\bar{h}$ and $\bar{h}$ will be smaller as $\bar{\mu}$ being greater with the same $\bar{W}$. We can get the relationship on $\bar{W}$ and $\bar{K}$. Thickness ratio of oil film $\bar{h}$ versus pressure ratio $\bar{P}_{r}$ for different pad restriction constant using three kinds pressure compensation methods as shown in Fig. 3.

In Fig. 2, the film thickness increases as viscosity ratio decreasing with the same load. This means that cooling lubricant at bearing pad makes film viscosity greater than supply oil viscosity will increase the film thickness also increase the load capacity, but the film stiffness increases as the viscosity ratio increasing. On the contrary, because of the friction force of lubricant making the film temperature rising makes the film viscosity decreasing, while decreases the film thickness but increases the film static stiffness. In Fig. 3, we can find the restriction constant is an important design 
parameter for the film thickness and load capacity when viscosity is constant. The film thickness and the load capacity both are inversely proportional to the restriction constant.

\section{Conclusions}

This study analyses the influences of thickness ratio of oil film, lubricant viscosity ratio, and restriction constant on its performances: load capacity and film static stiffness coefficient, and concludes as below: (1) When the viscosity ratio of lubrication fluid in restrictor stage to bearing recess stage is increasing, the load capacity of hydrostatic bearing will cause a certain amount of decrease, but its amplitude will reduce as the restriction constant increasing. (2) The viscosity ratio of lubrication fluid in restrictor stage to bearing recess stage can enhance the film static stiffness of hydrostatic bearing effectively, especially when greater than 1. (3) When the viscosity ratio of lubrication fluid in restrictor stage to bearing recess stage and restriction constant are increasing, the variation of thickness ratio of oil film will be stable more which mean having the good static stiffness. (4) Comparing three types of constant compensations in hydrostatic bearing, the constant flow has the best static performances, followed by the orifice restrictor, then the capillary restrictor.
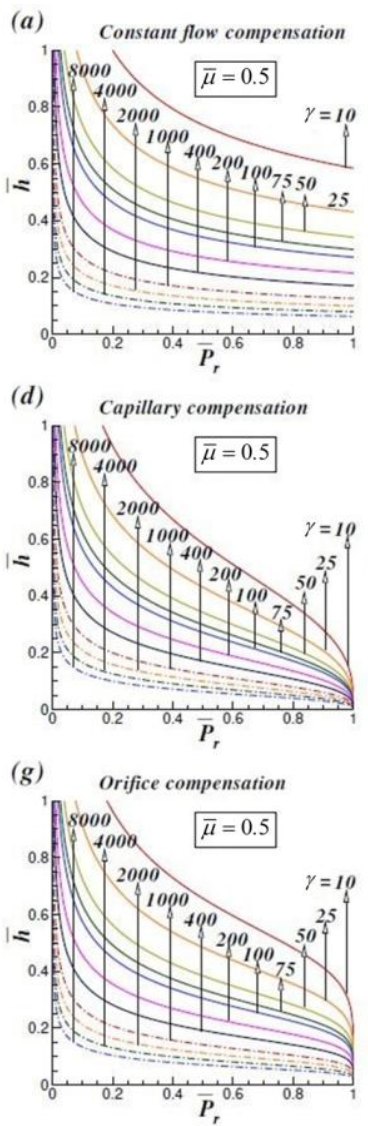

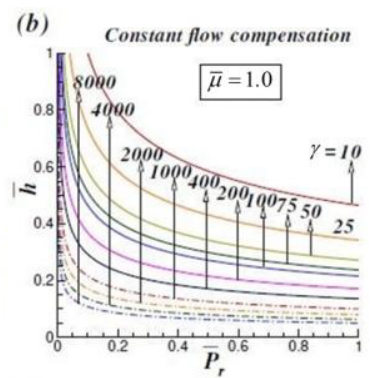

(e)

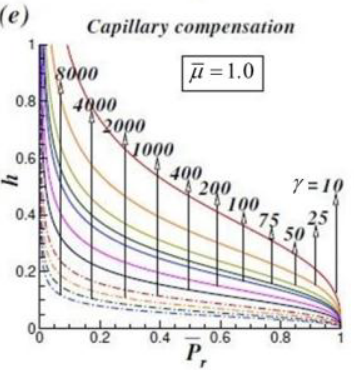

(h)
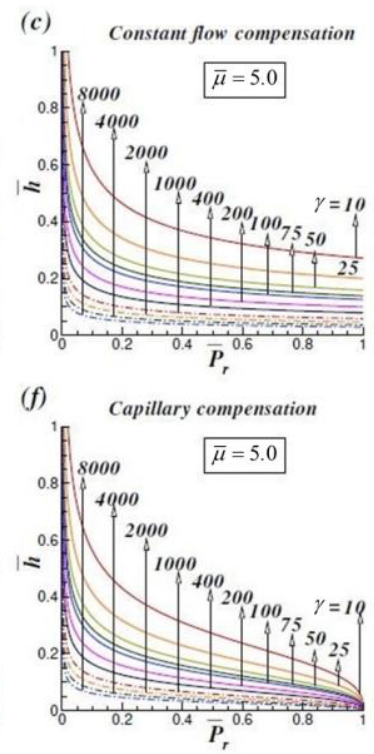

(i)
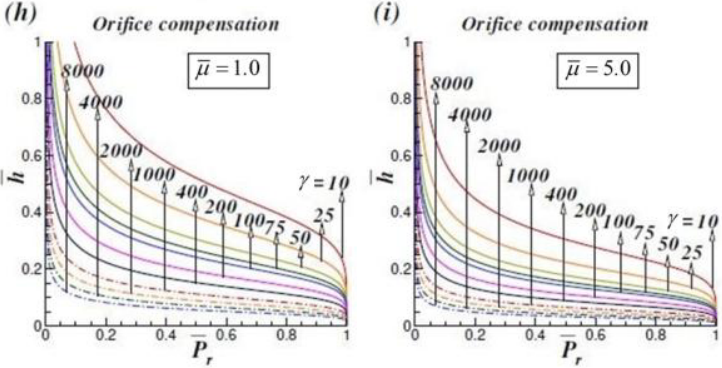

Figure 3. Thickness ratio of oil film $\bar{h}$ versus pressure ratio $\bar{P}_{r}$ for different pad restriction using constant flow compensation [(a)-(c)], capillary compensation [(d)-(f)] and orifice compensation $[(\mathrm{g})-(\mathrm{i})]$.

\section{Acknowledgement}


The authors greatly appreciate the support of this work by Grants NSC 103-2221-E-003-016-MY3 from the National Science Council Taiwan R. O. C.

\section{References}

1. Malanoski S.B., Loeb A.M., The effect of the method compensation on hydrostatic bearing stiffness, J Basic Eng-T ASME, 83(2), 179-187 (1961)

2. Wang D., Hydrostatic slideway and its design research, LUBR ENG, 4, 114-118 (2004)

3. Kang Y., Lee J.L., Huang H.C., Lin C.Y., Lee H.H., Peng D.X., Design for static stiffness of hydrostatic plain bearings: constant compensations, Ind Lubr Tribol, 63(3), 178-191 (2011)

4. Nikolakopoulos P.G., Papadopoulos C.A., Controllable high speed journal bearings, lubricated with electro-rheological fluids. An analytical and experimental approach, Tribol Int, 31, 225-234 (1998)

5. Bouzidane A., Thomas M., An electrorheological hydrostatic journal bearing for controlling rotor vibration, Comput Struct, 86, 463-472 (2008)

6. Bouzidane A., Thomas M., Nonlinear dynamic behavior of a flexible shaft supported by smart hydrostatic squeeze film dampers, J Tribol, 135, 031701-1 9 (2013)

7. Yu X.D., Fu X., Meng X.L., Liu D., Zhang Y.Q., Wang W.S., Experimental and numerical study on the temperature performance of high-speed circular hydrostatic thrust bearing, J Comput Theor Nanosci, 12(8), 1540-1545(6) (2015). 